Skin

Appendage

Disorders
Skin Appendage Disord 2017;3:222-224

DOI: 10.1159/000477415
Received: May 5, 2017

Accepted: May 8, 2017

Published online: June 17, 2017

\title{
Syphilitic Alopecia: Case Reports and Trichoscopic Findings
}

\author{
Isabella Doche ${ }^{a}$ Maria K. Hordinsky ${ }^{b}$ Neusa Y.S. Valente ${ }^{a}$ Ricardo Romiti $^{a}$ \\ Antonella Tostic

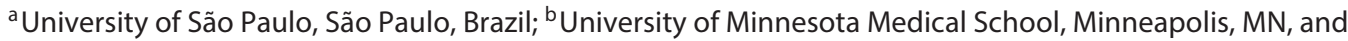 \\ 'University of Miami, Miller Medical School, Miami, FL, USA
}

\section{Established Facts}

- Syphilis can cause patchy or diffuse nonscarring hair loss.

- Alopecia can be the sole manifestation of the disease.

\section{Novel Insights}

- This is the first time zigzag hairs are described in the setting of syphilitic alopecia.

- Trichoscopy may help distinguish syphilitic alopecia from other causes of hair loss, especially in the absence of clinical lesions.

\section{Keywords}

Alopecia $\cdot$ Trichoscopy $\cdot$ Syphilis $\cdot$ Alopecia areata .

Dermoscopy $\cdot$ Hair

\begin{abstract}
There has been a resurgence of syphilis in the last decade. Although alopecia is not a common manifestation of the disease, the "moth-eaten" pattern is considered patognomonic. However, diffuse hair loss, termed essential alopecia, sometimes can be the only sign of syphilis infection and mimic other conditions, such as alopecia areata and telogen effluvium. We describe three patients with syphilis in whom the pattern of alopecia was not typical. Although clinical, histopathological, and trichoscopic examinations may share some similar findings with other hair diseases, the inclusion of syphilis in the differential diagnosis of hair loss causes is fundamental to a correct screening and diagnosis.
\end{abstract}

(c) 2017 S. Karger AG, Basel
(C) 2017 S. Karger AG, Basel

\section{KARGER}

E-Mail karger@karger.com

www.karger.com/sad

\section{Introduction}

Syphilis has become widespread worldwide with resurgence of new cases in the last decade. Alopecia is an uncommon clinical manifestation of secondary syphilis, ranging from 2.9 to $7 \%$ [1]. Two basic patterns of syphilitic alopecia (SA) have been described in the literature, symptomatic and essential. The first type presents with either a patchy or diffuse scalp alopecia associated with typical skin lesions of secondary syphilis. The "moth-eaten" pattern is the most common type of scalp alopecia and is considered to be a patognomonic manifestation of secondary syphilis [1-3]. Essential alopecia is extremely rare and presents as a unique clinical presentation of the disease. However, it appears as a nonscarring hair loss which can occasionally affect hair-bearing areas other than the scalp $[1,2]$ and mimic telogen effluvium and alopecia areata $[3,4]$. Even though the clinical aspects of

Isabella Doche, $\mathrm{MD}, \mathrm{PhD}$

Rua Cubatao 86 cj 1302 Vila Mariana

São Paulo, SP 01321001 (Brazil)

E-Mail isabelladoche@gmail.com 


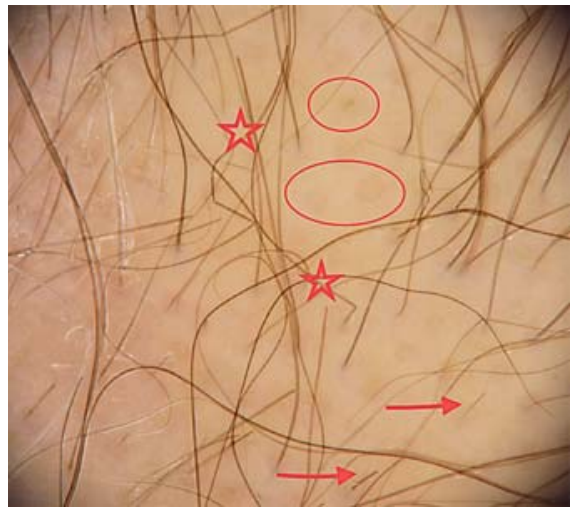

Fig. 1. Syphilitic alopecia. Decreased hair density, yellow dots (circles), broken hairs (arrows), and zigzag hairs (stars).

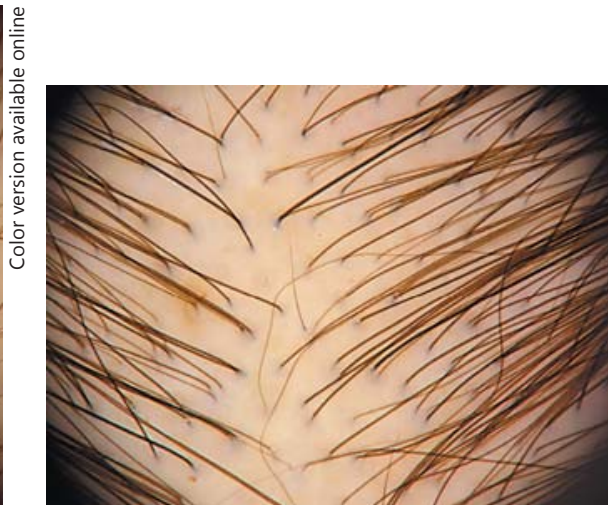

Fig. 2. Note complete hair regrowth after treatment.

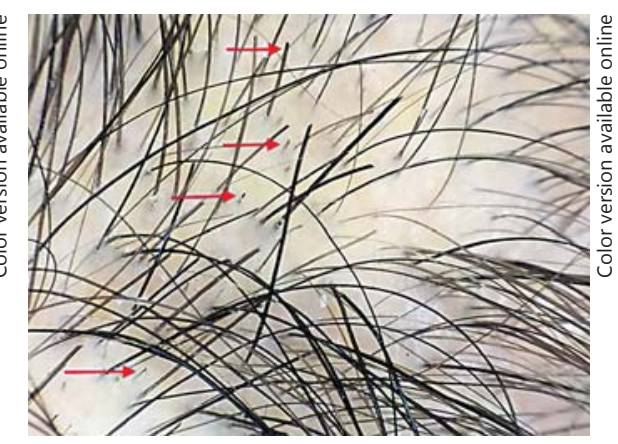

Fig. 3. Hair shaft variability and broken hairs (arrows) of different length, but no yellow dots or exclamation mark hairs.
SA are well described, the trichoscopic findings are not commonly reported in the literature $[5,6]$. We describe three cases of SA and their corresponding trichoscopic findings.

\section{Case Reports}

Case 1

A 51-year-old Caucasian man presented with a 3-month history of scalp hair shedding. The patient had been treated with topical steroids and 5\% minoxidil lotion with no improvement. Physical examination revealed diffuse nonscarring scalp alopecia mainly over the parietal, vertex, and occipital areas, associated with eyebrow and extremity hair loss. No patches of alopecia elsewhere or other skin lesions were noted. Scalp hair was thin and had a rough texture. Pull test was positive for telogen and anagen dystrophic hairs. Trichoscopy showed decreased hair density, yellow dots, broken hairs, and zigzag hairs (Fig. 1). Blood tests were within normal limits. Serological screening for syphilis showed increased titers for the Venereal Disease Research Laboratory (VDRL) test (1:8), and fluorescent treponemal antibody absorption was also positive. Histological examination from the affected parietal area showed decreased hair density, irregular hair shafts, and normal terminal/velus ratio (9:1) with moderate periinfundibular inflammatory lymphocytic infiltrate and hypoplastic sebaceous glands. Direct immunofluorescence demonstrated homogeneous, continuous, and mild deposition of IgG in the follicular basal membrane zone, C3 on the follicular epithelium, and IgG and C3 on the epidermal keratinocyte nuclei. Liquor analysis excluded neurolues. Immunohistochemistry with Treponema pallidum staining was negative in the hair shafts. After the use of erythromycin $500 \mathrm{mg}$ b.i.d. for 30 days, the patient presented with complete hair regrowth. VDRL was negative after 6 months and dermoscopy did not show any specific findings after the therapy (Fig. 2).

Syphilis and Trichoscopy
Case 2

A 32-year-old Caucasian man presented with diffuse scalp hair loss associated with irregular alopecia areata-like patches, and palmoplantar lesions. Trichoscopy showed hair shaft variability and broken hairs of different length, but no yellow dots or exclamation mark hairs (Fig. 3). The patient had complete regrowth after treatment with penicillin.

\section{Case 3}

A 41-year-old African-American man was referred with patchy scalp alopecia of 2 -week duration resembling alopecia areata. A maculopapular rash involving the trunk and oral lesions could also be noted. Broken hairs were seen on trichoscopy. None of the patients complained of scalp itching or trichodynia.

\section{Discussion}

The precise pathogenesis for SA still has to be ascertained. However, the immune response to T. pallidum, or its presence, could be related to the loss of terminal hairs, hair follicle cycle arrest, and hair bend resulting in empty follicles and broken hairs. Diffuse SA can mimic alopecia areata on both clinical and histopathological examinations $[3,4]$; however, noninflammatory biopsies can be misdiagnosed as telogen effluvium [7]. Although zigzag hairs can often be seen in patients with tinea capitis [8], it is not patognomonic of a fungal infection. Trichotillomania and systemic lupus must also be ruled out. In our cases, although clinical lesions were not diagnostic, serological tests led to the correct diagnosis and complete hair regrowth was achieved after antibiotic therapy. Clinical, histopathological, and trichoscopic evaluations may help distinguish SA from other causes of hair loss, especially when typical skin lesions of syphilis are absent.

Skin Appendage Disord 2017;3:222-224 


\section{Statement of Ethics}

The authors state that all subjects have given their informed consent for the pictures and the study has been approved by the ethics committee of the University of São Paulo.

\section{Disclosure Statement}

The authors declare no conflict of interest and no funding sources for this paper.

\section{References}

1 Vafaie J, Weinberg JM, Smith B, et al: Alopecia in association with sexually transmitted disease: a review. Cutis 2005;76:361-366.

2 Cuozzo DW, Benson PM, Sperling LC, et al: Essential syphilitic alopecia revisited. J Am Acad Dermatol 1995;32:840-843.

3 Bi MY, Cohen PR, Robinson FW, et al: Alopecia syphilitica - report of patient with secondary syphilis presenting as moth-eaten alopecia and a review of its common mimickers. Dermatol Online J 2009;15:6.
4 Lee JW, Jang WS, Yoo KH, et al: Diffuse pattern essential syphilitic alopecia: an unusual form of secondary syphilis. Int J Dermatol 2012;51:1006-1007.

5 Ye Y, Zhang X, Zhao Y, Gong Y, Yang J, Li H, Zhang X: The clinical and trichoscopic features of syphilitic alopecia. J Dermatol Case Rep 2014;8:78-80.
6 Piraccini BM, Broccoli A, Starace M, Jasper V, D'Antuono A, Dika A, Patrizi A: Hair and scalp manifestations in secondary syphilis: epidemiology, clinical features and trichoscopy. Dermatology 2015;231:171-176.

7 Sperling LC, Cowper SE, Knopp EA: An Atlas of Hair Pathology with Clinical Correlations. Second edition. United Kingdom, Informa, 2012.

8 Elghblawi E: Idiosyncratic findings in trichoscopy of tinea capitis: comma, zigzag hairs, corkscrew, and morse code-like hair. Int J Trichology 2016;8:180-183. 